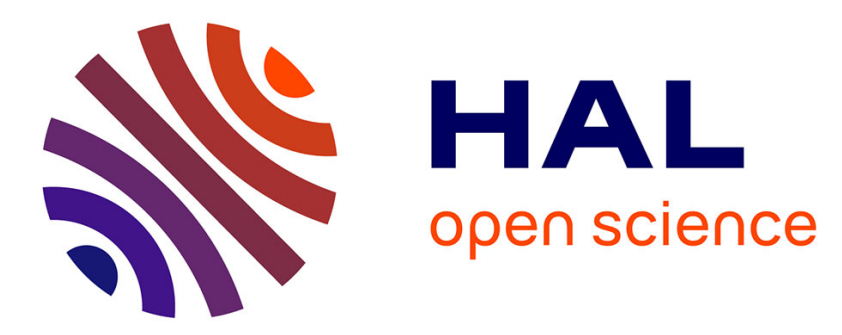

\title{
Large spin splittings due to the orbital degree of freedom and spin textures in a ferroelectric nitride perovskite
} Hong Jian Zhao, Peng Chen, Charles Paillard, Rémi Arras, Yue-Wen Fang, Xu Li, Julien Gosteau, Yurong Yang, Laurent Bellaiche

\section{- To cite this version:}

Hong Jian Zhao, Peng Chen, Charles Paillard, Rémi Arras, Yue-Wen Fang, et al.. Large spin splittings due to the orbital degree of freedom and spin textures in a ferroelectric nitride perovskite. Physical Review B, 2020, 102 (4), pp.041203. 10.1103/PhysRevB.102.041203 . hal-02988959

\section{HAL Id: hal-02988959 https://hal.science/hal-02988959}

Submitted on 21 Nov 2020

HAL is a multi-disciplinary open access archive for the deposit and dissemination of scientific research documents, whether they are published or not. The documents may come from teaching and research institutions in France or abroad, or from public or private research centers.
L'archive ouverte pluridisciplinaire HAL, est destinée au dépôt et à la diffusion de documents scientifiques de niveau recherche, publiés ou non, émanant des établissements d'enseignement et de recherche français ou étrangers, des laboratoires publics ou privés. 


\title{
Large spin splittings due to the orbital degree of freedom and spin textures in a ferroelectric nitride perovskite
}

\author{
Hong Jian Zhao $\odot,{ }^{1, *}$ Peng Chen,,${ }^{1, \dagger}$ Charles Paillard $\odot,{ }^{2}$ Rémi Arras, ${ }^{3}$ Yue-Wen Fang $\odot,{ }^{4}$ Xu Li,${ }^{5}$ Julien Gosteau, ${ }^{3}$ \\ Yurong Yang, ${ }^{5}$ and Laurent Bellaiche ${ }^{1, \$}$ \\ ${ }^{1}$ Physics Department and Institute for Nanoscience and Engineering, University of Arkansas, Fayetteville, Arkansas 72701, USA \\ ${ }^{2}$ Laboratoire SPMS, CentraleSupélec/CNRS UMR8580, Université Paris-Saclay, 8-10 Rue Joliot-Curie, 91190 Gif-sur-Yvette, France \\ ${ }^{3}$ CEMES, Université de Toulouse, CNRS, UPS, 29 Rue Jeanne Marvig, F-31055, Toulouse, France \\ ${ }^{4}$ Department of Materials Science and Engineering, Kyoto University, Kyoto 606-8501, Japan \\ ${ }^{5}$ National Laboratory of Solid State Microstructures, Department of Materials Science and Engineering, \\ Nanjing University, Nanjing 210093, China
}

(Received 10 December 2019; revised 24 March 2020; accepted 29 June 2020; published 20 July 2020)

\begin{abstract}
First-principles simulations are conducted to predict that ferroelectric nitride perovskite $\mathrm{LaWN}_{3}$ not only exhibits large spin splittings $(2.7 \mathrm{eV} \AA$ ) but also possesses unique spin textures for some of its conduction levels. Such spin splittings around the $\Gamma$ and $L$ points cannot be interpreted as a typical mixture of Rashba or Dresselhaus configurations but rather require the development of four-band $\boldsymbol{k} \bullet \boldsymbol{p}$ models with high-order terms. We further identify that, for some bands, spin splittings can be greatly contributed by the pure orbital degree of freedom (PODF), a unique character of our four-band Hamiltonian compared to the traditional two-band version. The concept of PODF-enhanced spin splittings paves a way for designing materials with large spin splittings. Moreover, the energy levels possessing such large splittings and complex spin textures can be brought close to the conduction-band minimum by applying epitaxial strain.
\end{abstract}

DOI: 10.1103/PhysRevB.102.041203

\section{INTRODUCTION}

Spin splitting in ferroelectric materials is attracting a great deal of attention because it offers the possibility of novel spintronic devices based on electric control of spin textures [1-15]. Recently, various ferroelectrics presenting large spin splittings have been proposed theoretically or synthesized, including GeTe [6], SnTe [7], hyperferroelectrics [10,12], halides [8], and iodide perovskites [11], and the recently discovered $\mathrm{Bi}_{2} \mathrm{WO}_{3}$ [3], $\mathrm{BiInO}_{3}$ [5], and $\mathrm{HfO}_{2}$ [9]. Among them, SnTe [7] and GeTe [6] have the two largest spin-splitting parameters (6.8 and $4.8 \mathrm{eV}$. $\AA$ ). Moreover, most of the observed spin textures, including those for $\mathrm{HfO}_{2}$ [9] and $\mathrm{BinO}_{3}$ [5], are of linear Dresselhaus (spin-splitting parameter denoted by $\lambda_{\mathrm{D}}$ ) or linear Rashba (spin-splitting parameter referred to as $\lambda_{R}$ ) type, or even a mixture of them by varying the ratio between $\lambda_{D}$ and $\lambda_{R}$. For instance, the persistent spin textures, whose spin configurations do not depend on momentum, have been predicted to occur in $\mathrm{BiInO}_{3}$ [5], $\mathrm{CsBiNb}_{2} \mathrm{O}_{7}$ [13], and other materials [5] (in these cases, $\lambda_{\mathrm{D}}$ and $\lambda_{\mathrm{R}}$ are almost equal in magnitude). On one hand, the spin splittings and textures for most of these systems were well described by a two-band effective Hamiltonian involving only spin degree of freedom (SDF) (i.e., spin up $|\uparrow\rangle$ and spin down $|\downarrow\rangle$ ). However, one should also realize that the pure orbital degree

\footnotetext{
*hz001@uark.edu

†pc011@uark.edu

‡laurent@uark.edu
}

of freedom (PODF)-for example, the double-degenerate ( $|x\rangle$, $|y\rangle$ ) states in $C_{3 v}$ point group-may contribute to spin splittings. More explicitly, in a nonmagnetic system without spin-orbital coupling (SOC), all symmetry-protected degenerate levels are referred to as PODF. Note that incorporating both SDF and PODF will result in higher "dimensional" Hamiltonian (e.g., with the basis of $|x \uparrow\rangle,|y \uparrow\rangle,|x \downarrow\rangle,|y \downarrow\rangle)$ compared to cases with SDF only (e.g., with the basis of $|\uparrow\rangle$ and $|\downarrow\rangle$ ), possibly leading to undiscovered complex and important features such as PODF-contributed spin splittings and novel spin textures (i.e., beyond the linear superposition of Dresselhaus- and Rashba types, and beyond two-band models). If this is the case, the PODF-reinforced large spin splittings and possible interesting spin textures may greatly enhance the opportunity to design new materials with large spin splittings. Such a corresponding concept regarding the contribution of PODF to spin splittings, which has been implied in literature [5,9] but not extensively noticed, is also of fundamental interest.

To this end, we decided to investigate a compound, namely nitride $\mathrm{LaWN}_{3}$ perovskite that has been recently predicted to be a ferroelectric semiconductor with $R 3 c$ space group [16-18] and polarization of $\sim 61 \mu \mathrm{C} / \mathrm{cm}^{2}$ along the pseudocubic $\langle 111\rangle$ direction [18]. One reason to tackle this system is that it contains the $5 d$ element $\mathrm{W}$ whose SOC is expected to be strong, probably leading to large spin splittings at some conduction bands. Other reasons are (i) at the $\Gamma$ point, there are twofold degenerate PODF represented by $(|x\rangle,|y\rangle)$ states; and (ii) the existence of nonprimitive lattice translations of $R 3 c$ space group may enforce extra "orbital" degeneracy (e.g., additional sublattice degrees of freedom [5,9], serving 
as PODF) at some high-symmetry $k$ points-a well-known character of nonsymmorphic space group $[19,20]$. Items (i) and (ii) allow us to investigate if PODF can induce large spin splittings and novel spin textures. Last but not least, ternary nitride compounds have started to receive theoretical [16] and experimental [21] attention; a recent study by Zakutayev and coworkers suggests the possibility of growing perovskite $R 3 c \mathrm{LaWN}_{3}$-based materials starting from $\mathrm{LaWN}_{3-\delta} \mathrm{O}_{\delta}$ and reducing $\delta$ [22] and perovskite $\mathrm{LaWN}_{3}$ was soon synthesized and identified with $R 3 c$ symmetry [23]. In the present work, we demonstrate that $\mathrm{LaWN}_{3}$ has not only PODF-contributed spin splittings at the $\Gamma$ and $L$ points but also unusual spin textures. In addition, large spin splittings are predicted for some of these conduction levels, with these latter levels being even controllable by epitaxial strain.

\section{METHODS}

Our methods are described in the Supplemental Material (SM) [24] (see the Supplemental Material references [25-37] as well). We carried out density-functional theory (DFT) simulations using VASP code $[38,39]$, and conducted symmetry analysis using Ref. [40], Bilbao crystallographic server [41,42], and SEEK-PATH software [43]. Unless mentioned, our results are based on the PBEsol (Perdew-Burke-Ernzerhof revised for solids) functional [44]. Since $\mathrm{LaWN}_{3}$ is nonmagnetic, we have not artificially initialized spin configurations; the final spin configurations are determined by VASP after fully converging the electronic self-consistent loop. The basic spirit to compute the spin textures (from eigenvectors) is given in Sec. I.2.4 of SM [24].

\section{RESULTS AND DISCUSSION}

Band structures, as well as density of states, are summarized in Fig. S4 and discussed in Sec. III.1 of SM [24]. Band splittings along the $\Gamma-L$ line via PODF are shown in Fig. 1(a) (i.e., without SOC); meanwhile, the band splittings incorporating both PODF and SDF (termed as spin splittings) are displayed in Fig. 1(b) (i.e., with SOC). Using the simple expression $E=E_{0} \pm \lambda_{S} \Delta k+b(\Delta k)^{2}$ we fit the energy levels around the $L(L-\Gamma$ line $), \Gamma$ ( $\Gamma-L$ line $)$, and $F\left(F-S_{2}\right.$ line $)$ points and obtain the following spin-splitting parameters for $R 3 c \mathrm{LaWN}_{3}$ bulk: $\sim 2.7$ and $\sim 1.6 \mathrm{eV} \AA$ for bands around $L$ [A and $\mathrm{B}$ in Fig. 1(b)], $\sim 0.13$ and $\sim 0.08 \mathrm{eV} \AA$ for bands around $\Gamma$ [C and D in Fig. 1(b)]. Around the $F$ point, the spin splitting is $\sim 0.8 \mathrm{eV} \AA$ (e.g., along the $F-S_{2}$ line). Strikingly, the spin splitting around the $L$ point $[\sim 2.7 \mathrm{eV} \AA$, see A in Fig. 1(b)] exceeds most of those reported in the literature (e.g., 1.2-2.2 eV $\AA$ for $\mathrm{BiTeCl}[45,46], \sim 2 \mathrm{eV} \AA$ for $\mathrm{BiTeBr}[46]$, $\sim 0.6 \mathrm{eV} \AA$ for $\mathrm{HfO}_{2}$ [9], $\sim 2.6 \mathrm{eV} \AA$ for $\mathrm{NaZnSb}[12]$, and $1.9 \mathrm{eV}$. $\AA$ for $\mathrm{BiInO}_{3}$ [5]), even if it is lower than some of the highest values $(3.9-4.3 \mathrm{eV}$. $\mathrm{A}$ for BiTeI [46,47], $4.8 \mathrm{eV} \AA$ for GeTe [6], and $6.8 \mathrm{eV} \AA$ for SnTe [7]). The valence-band maximum (VBM) and conduction-band minimum (CBM) are in the vicinity of the $F$ and $\Gamma$ points, respectively, with a band gap of $\sim 0.85 \mathrm{eV}$ (with SOC). In Sec. III.1, our calculations indicate that the band gap at PBEsol [44] level is underestimated by $\sim 1 / 2$ than that at HSEsol (improved hybrid functional for solids) [48] level.
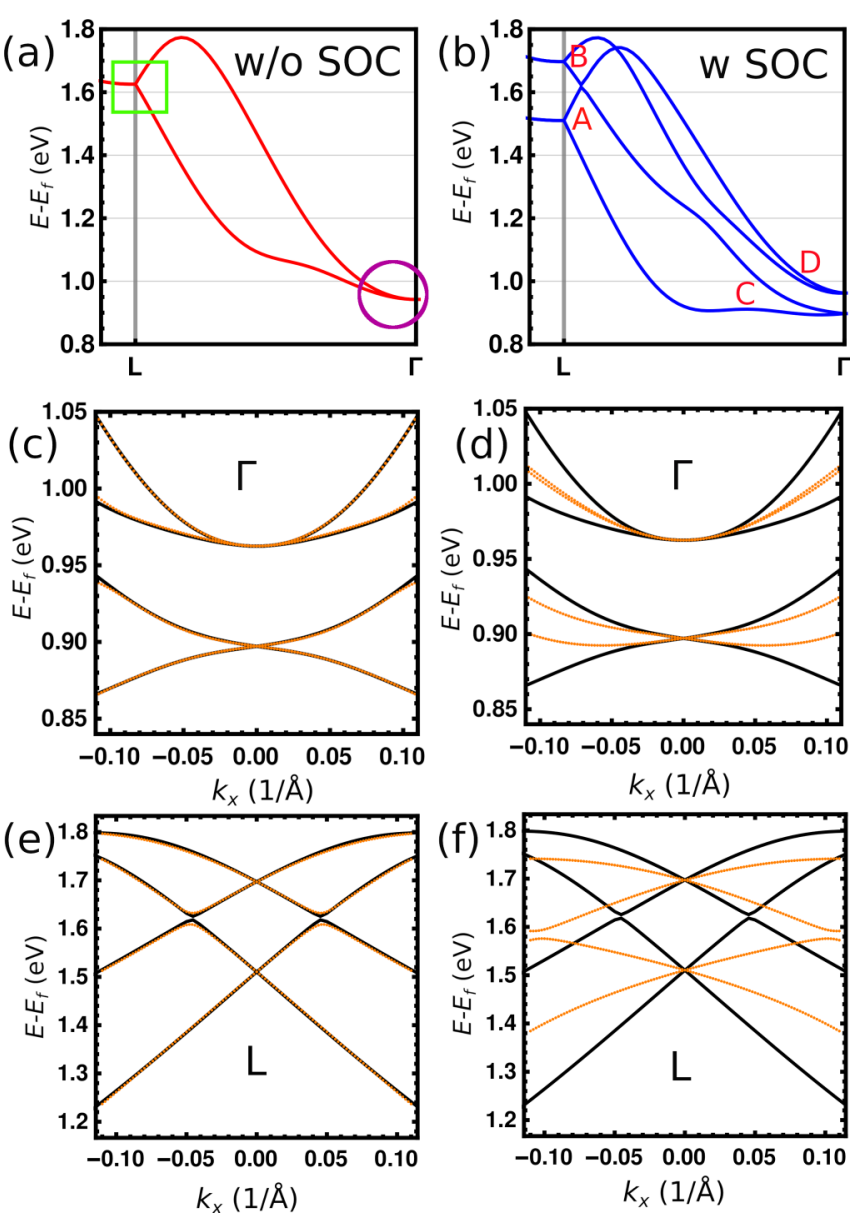

FIG. 1. Conduction bands of $\mathrm{LaWN}_{3}$ along the $L-\Gamma$ line for cases without SOC (a) and with SOC (b). In (b), we use "A," "B," "C," and "D" to mark the spin splittings around the $L$ and $\Gamma$ points. (c), (e) Conduction-band structures (energy versus $k_{x}$, with SOC) around the $\Gamma$ and $L$ points, as obtained by DFT (black lines) and full models (orange dots) described in the text. Note that the $k_{x}$ direction is not along the $L-\Gamma$ line (See Fig. S3 and Sec. I.1 of SM [24]). The parametrization of the models for $\Gamma$ and $L$ points is indicated in Sec. III.2 of SM [24]. (d), (f) Conduction-band structures around the $\Gamma$ and $L$ points obtained from DFT (black lines) and modified models (orange dots). By modified models, we mean that $\eta$, $\xi$ in Eq. (1), as well as $\omega, v, \eta, U, V$ in Eq. (2) are all set to zero, while the remaining model parameters remain identical to those used in the full models.

As also indicated in the SM [24] (see Sec. III.3), the spin textures of the aforementioned conduction levels at the $F$ point are rather trivial. Indeed, such spin textures are well captured by a two-band model with linear-in- $k$ spin-splitting terms and thus are similar to those of the various ferroelectric Rashba/Dresselhaus systems mentioned in our Introduction. We thus concentrated on the conduction levels for the $\Gamma$ and $L$ points, which have rather unusual spin textures [Figs. 2(a)2(h)]. They indeed appear to deviate from simple solutions typically obtained by two-band $\boldsymbol{k} \bullet \boldsymbol{p}$ model, such as those associated with Rashba and/or Dresselhaus types. We are not aware that such complex spin textures have been previously reported. Such complexity for the spin textures around the $\Gamma$ and $L$ points may be due to the fact that SDF and PODF are "coupled together." To check such a possibility, we decided 

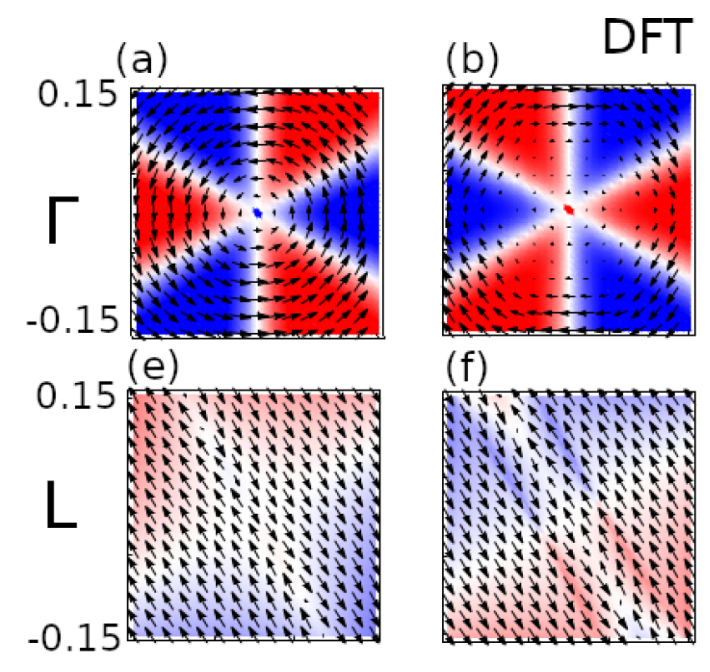

(f)
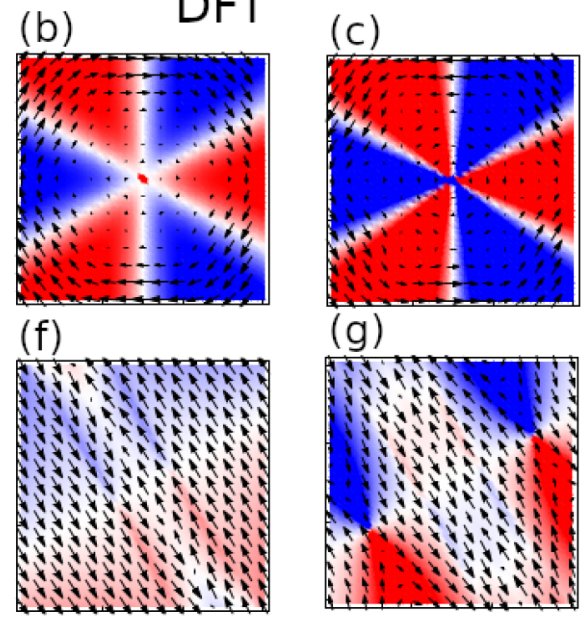

(g)
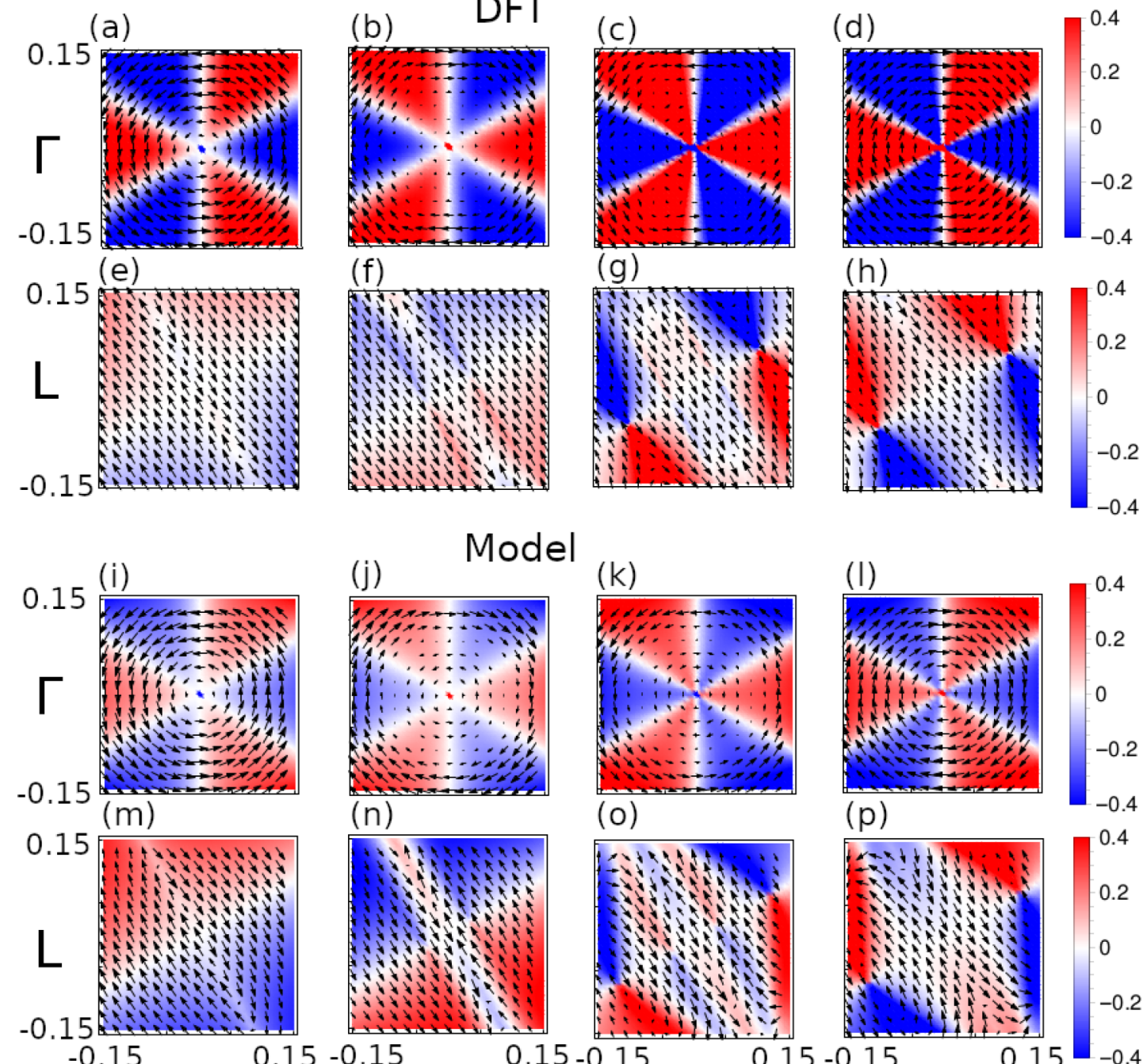

(n)

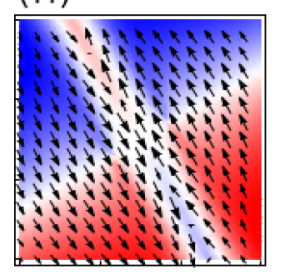

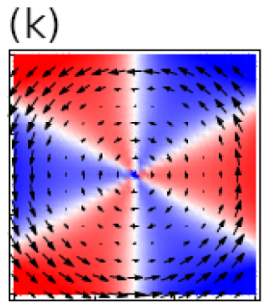

(o)

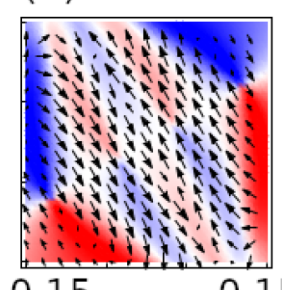

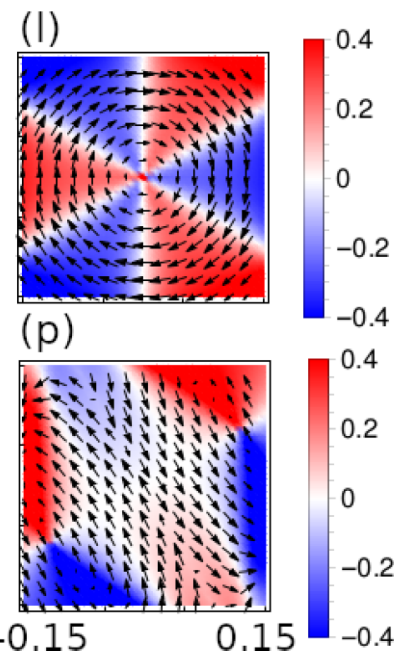

FIG. 2. Spin textures of the lowest four conduction levels for $R 3 c \mathrm{LaWN}_{3}$ bulk around the $\Gamma[(\mathrm{a})-(\mathrm{d})]$ and $L[(\mathrm{e})-(\mathrm{h})]$ points obtained by DFT. (i)-(p) Counterparts of (a)-(h) obtained by four-band models [e.g., Eq. (1) for (i)-(l) and Eq. (2) for (m)-(p)]. The arrows and color bars denote the in-plane and out-of-plane components of spin textures with respect to the $\left(k_{x}, k_{y}\right)$ plane. The units of $k_{x}$ (horizontal axis) and $k_{y}$ (vertical axis) are $\AA^{-1}$. Our spin textures are prepared using the following convention [(a)-(d) as examples]: given that at each ( $\left.k_{x}, k_{y}\right)$ point, the corresponding energy eigenvalues associated with panels (a), (b), (c), and (d) are $e_{1 k}, e_{2 k}, e_{3 k}, e_{4 k}$, respectively; then $e_{1 k} \leqslant e_{2 k} \leqslant e_{3 k} \leqslant e_{4 k}$ holds.

to construct high-order four-band $\boldsymbol{k} \bullet \boldsymbol{p}$ models for the $\Gamma$ and $L$ points. The details about constructing such models are discussed in Sec. I.2 of the SM [24]. In particular, this $\boldsymbol{k} \bullet \boldsymbol{p}$ model around the $\Gamma$ point provides a Hamiltonian of the form

$$
\begin{aligned}
H\left(k_{x}, k_{y}\right)= & E_{0}+\alpha\left(k_{x}^{2}+k_{y}^{2}\right)+\beta\left(k_{x}^{2}+k_{y}^{2}\right)^{2}+\delta\left(k_{x}^{2}+k_{y}^{2}\right)^{3} \\
& +\eta\left[\left(k_{x}^{2}-k_{y}^{2}\right) \gamma_{z}+2 k_{x} k_{y} \gamma_{x}\right]+\left[\Delta+\Lambda\left(k_{x}^{2}+k_{y}^{2}\right)\right. \\
& \left.+\mathrm{K}\left(k_{x}^{2}+k_{y}^{2}\right)^{2}\right] \gamma_{y} \sigma_{z}+\left[\kappa+\varsigma\left(k_{x}^{2}+k_{y}^{2}\right)\right] \\
& \times\left(k_{x} \sigma_{y}-k_{y} \sigma_{x}\right)+\lambda k_{x}\left(k_{x}^{2}-3 k_{y}^{2}\right) \sigma_{z} \\
& +\xi k_{x}\left(k_{x}^{2}-3 k_{y}^{2}\right) \gamma_{y}+\rho\left[\left(k_{x} \gamma_{x}-k_{y} \gamma_{z}\right) \sigma_{x}\right. \\
& \left.-\left(k_{y} \gamma_{x}+k_{x} \gamma_{z}\right) \sigma_{y}\right]
\end{aligned}
$$

where both $\gamma_{i}$ and $\sigma_{j}(i, j=x, y, z)$ are Pauli matrices denoting orbital and spin degrees of freedom, respectively. $\gamma_{i} \sigma_{j}$ is interpreted as the Kronecker product of $\gamma_{i}$ and $\sigma_{j}\left(\gamma_{i} \otimes \sigma_{j}\right)$; $\gamma_{i}$ and $\sigma_{i}$ denote $\gamma_{i} \otimes \sigma_{1}$ and $\gamma_{1} \otimes \sigma_{i}\left(\gamma_{1}\right.$ and $\sigma_{1}$ being $2 \times 2$ identity matrix). Note that $\eta$, $\xi$ terms in Eq. (1) are related to PODF (contributed only by orbital degree of freedom). Similarly, one can construct the following four-band model for the $L$ point:

$$
\begin{aligned}
H\left(k_{x}, k_{y}\right)= & E_{0}+\alpha\left(k_{x}^{2}+\sqrt{3} k_{x} k_{y}\right)+\beta\left(k_{y}^{2}-\sqrt{3} k_{x} k_{y}\right) \\
& +R\left(k_{x}^{2}+\sqrt{3} k_{x} k_{y}\right)^{2}+S\left(k_{y}^{2}-\sqrt{3} k_{x} k_{y}\right)^{2} \\
& +T\left(k_{x}^{2}+\sqrt{3} k_{x} k_{y}\right)\left(k_{y}^{2}-\sqrt{3} k_{x} k_{y}\right) \\
& +\left(\sqrt{3} k_{x}+k_{y}\right)\left(k_{x}-\sqrt{3} k_{y}\right)\left(\omega \gamma_{x}+v \gamma_{y}\right) \\
& +\eta\left(\sqrt{3} k_{x}+k_{y}\right) \gamma_{z}+\left[U\left(k_{x}^{2}+\sqrt{3} k_{x} k_{y}\right)\right. \\
& \left.+V\left(k_{y}^{2}-\sqrt{3} k_{x} k_{y}\right)\right]\left(\sqrt{3} k_{x}+k_{y}\right) \gamma_{z} \\
& +\left(\sqrt{3} k_{x}+k_{y}\right)\left(F \gamma_{x}+G \gamma_{y}\right) \sigma_{z}
\end{aligned}
$$




$$
\begin{aligned}
& +\mathrm{A}\left(\sqrt{3} k_{x}+k_{y}\right)\left(k_{x}-\sqrt{3} k_{y}\right) \gamma_{z} \sigma_{z} \\
& +\varsigma\left(k_{x}-\sqrt{3} k_{y}\right) \sigma_{z}+\rho\left(\sqrt{3} k_{x}+k_{y}\right)\left(\sqrt{3} \sigma_{y}-\sigma_{x}\right) \\
& +\mathrm{B}\left(\sqrt{3} k_{x}+k_{y}\right)^{2} \gamma_{z}\left(\sqrt{3} \sigma_{y}-\sigma_{x}\right) \\
& +\mathrm{M}\left(k_{x}-\sqrt{3} k_{y}\right)\left(\sqrt{3} \sigma_{x}+\sigma_{y}\right) \\
& +\Delta\left(\sqrt{3} k_{x}+k_{y}\right)\left(k_{x}-\sqrt{3} k_{y}\right) \gamma_{z}\left(\sqrt{3} \sigma_{x}+\sigma_{y}\right) \\
& +\varepsilon \gamma_{z}\left(\sqrt{3} \sigma_{y}-\sigma_{x}\right)+\left[\mathrm{O}\left(k_{x}^{2}+\sqrt{3} k_{x} k_{y}\right)\right. \\
& \left.+\mathrm{H}\left(k_{y}^{2}-\sqrt{3} k_{x} k_{y}\right)\right] \gamma_{z}\left(\sqrt{3} \sigma_{y}-\sigma_{x}\right) \\
& +\left(\sqrt{3} k_{x}+k_{y}\right)\left(\kappa \gamma_{x}+\chi \gamma_{y}\right)\left(\sqrt{3} \sigma_{x}+\sigma_{y}\right) . \\
& +\left(k_{x}-\sqrt{3} k_{y}\right)\left(\mathrm{K} \gamma_{x}+\mathrm{X} \gamma_{y}\right)\left(\sqrt{3} \sigma_{y}-\sigma_{x}\right)
\end{aligned}
$$

Note also that the $\omega, v, \eta, U, V$ terms in Eq. (2) denote the contribution from PODF. We then fit these models to DFT results and compute the model-based band structures and spin textures. The comparisons between DFT and models for the band structures and spin textures are shown in Fig. 1(c), Fig. 1(e), and in Figs. 2(a)-2(p). Our models based on Eqs. (1) and (2) can reproduce well the DFT results for the band structures (we only show in Figs. 1(c) and 1(e) the band structures along the $k_{x}$ direction; the detailed fittings are shown in Fig. S5 of the SM [24]) and spin textures around the $\Gamma$ and $L$ points. Without SOC [Fig. 1(a)], the conduction levels at the $\Gamma$ point (purple circle) are twofold degenerate, as similar to the levels at the $L$ point (green rectangle); along the $L-\Gamma$ line, splittings occur due to PODF instead of SDF (because we are at a spinless case). When being away from the $\Gamma$ and $L$ points, such PODF-contributed splittings are implied by the $\eta, \xi$ terms in Eq. (1) and by the $\omega, v, \eta, U, V$ terms in Eq. (2), respectively. However, one cannot obtain eigenvalues of Eqs. (1) and (2) simply in analytic forms, preventing the direct examination of PODF's effect on spin splittings. Alternatively, we decided to check it numerically: starting from the well-parametrized models, let us switch off the $\eta$, $\xi$ terms in Eq. (1) and $\omega, v, \eta, U, V$ terms in Eq. (2), and maintain the remaining parameters unchanged when computing the band structures along $k_{x}$ [see Figs. 1(d) and 1(f)]. Without including the PODF-related terms, the spin splittings around both the $\Gamma$ and $L$ points are significantly changed as compared to Figs. 1(c) and 1(d), respectively, which thus emphasizes the important role of PODF on spin splittings.

It is also interesting and important to know that we numerically find that these spin textures, unlike the band structure, cannot be correctly reproduced by the model if the high-order-in- $k$ terms (i.e., the terms involving $\zeta, \lambda$, $\xi$ for the $\Gamma$ point and the $\omega, v, U, V, \mathrm{~A}, \mathrm{~B}, \Delta, \mathrm{O}$, and $\mathrm{H}$ terms for $L$ ) are excluded from Eqs. (1) and (2)—as evidenced in Figs. S8 and S9 and in Sec. III.4 of the SM [24]. Without these high-order terms, the discrepancy between the model and DFT for the out-of-plane components is too large to be considered as numerical errors. Our models of Eqs. (1) and (2) for the $\Gamma$ and $L$ points contain some terms that have been previously proposed [4,5,9,13,14,49,50]. Recently, we also became aware of work about Rashba-Dresselhaus spin splittings in $\mathrm{LaWN}_{3}$ [51]. The authors discussed the spin splittings for $R 3 c \mathrm{LaWN}_{3}$ around the $\Gamma$ point as well but focused on the

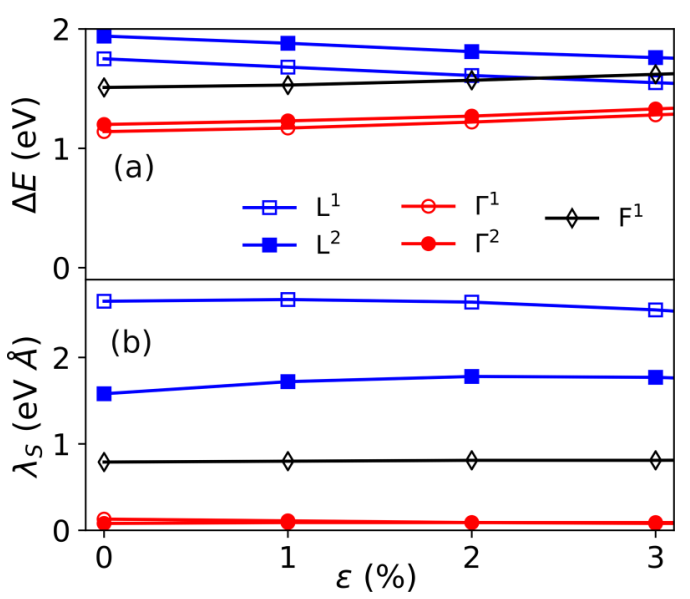

FIG. 3. (a) Energy of the conduction bands at the $\Gamma, L$, and $F$ points with respect to $E_{\Gamma 0}$ (the highest occupied valence-band level at the $\Gamma$ point, which is not the VBM). The superscripts 1 and 2 denote the lower and higher conduction levels around the $\Gamma, L$, and $F$ points. (b) The spin-splitting parameters of the corresponding conduction bands around the $\Gamma, L$, and $F$ points.

VBM which does not contain PODF and is captured well by a two-band model containing SDF only. Our discovered spin splittings and textures for conduction bands around the $\Gamma$ and $L$ points of $R 3 c \mathrm{LaWN}_{3}$ are quite unique since four-band models with significant squared- and/or cubic-in- $k$ terms are needed to reproduce spin splittings and textures, and since PODF reinforces spin splittings. Such uniqueness has rarely been mentioned in the literature for ferroelectric semiconductors. We also found that our DFT-determined spin textures at the $\Gamma, L$, and $F$ points are reversible by switching the polarization (see Sec. III.5 of the SM [24]), which is fully consistent with Ref. [8] demonstrating the universality of such reversibility based on symmetry arguments. We also determined the band structures, spin splittings, and textures by other functionals (e.g., PBE [52], PBE $+U$ [53], and SCAN [54]); the results from various functionals coincide with each other (e.g., the maximum variation is $\sim 10 \%$ for $L$-point's $\lambda_{S}$ ) (see Sec. III.6 of SM [24]).

One can also realize that the lowest conduction level at the $L$ point (which has the highest spin splitting) is not the CBM of $R 3 c \mathrm{LaWN}_{3}$ bulk. Such CBM is located in the vicinity of the $\Gamma$ point at $\sim 0.85 \mathrm{eV}$ above the valence-band maximum, and is lower by $\sim 0.66 \mathrm{eV}$ from the lowest conduction level at the $L$ point. However, it is known that strain can tune band-energy levels in semiconductors [55]. We thus decided to explore the effect of misfit strain $(\varepsilon)$ within (111) plane on spin splittings and energy levels for the $\Gamma, L$, and $F$ points of $R 3 c \mathrm{LaWN}_{3}$ (such strain can practically arise by growing $\mathrm{LaWN}_{3}$ films on top of cubic (111) or hexagonal (0001) substrates [56]). Figure 3 reports information about the band structures and spin splittings for $R 3 c \mathrm{LaWN}_{3}$ film under epitaxial tensile strain $\varepsilon$; note that $R 3 c$ phase is stable for $\varepsilon<3 \%$ (see Fig. S15 in the SM [24]). The $L^{1}$ and $L^{2}$ levels become closer in energy to the conduction levels indexed by the $\Gamma$ point as the $\varepsilon$ strengthens, and the spin-splitting parameters are rather insensitive to $\varepsilon$ (we also found that the polarization of $R 3 c$ phase is insensitive to $\varepsilon$ : between 0 
and $3 \%$ strain, the polarization is $61.5 \pm 1.5 \mu \mathrm{C} / \mathrm{cm}^{2}$, as predicted by Berry-phase method [57]. The barrier to switch the polarization (through $R-3 c$ phase) is $\sim 110 \mathrm{meV} /$ f.u., lower than $\sim 130 \mathrm{meV} /$ f.u. of $R 3 c \mathrm{LiNbO}_{3}$ [58]). Such features are promising to take advantage of the large spin splittings at the $L$ point, by making the lowest unoccupied levels of the $L$ point closer to the CBM of the system. One can also grow $\mathrm{LaWN}_{3}$ on metals whose work function or electronic affinity allows one to directly access states which are neither CBM or VBM [59] (e.g., the $L^{1}$ levels in Fig. 3). Furthermore, one can readily incorporate strain into our models to interpret its effect on band structures in a phenomenological way. Under epitaxial tensile strain $\left(\varepsilon_{x x}=\varepsilon_{y y}=\varepsilon\right)$, the coefficients of Eqs. (1) and (2) should be renormalized. For instance, $K\left(k_{x} \sigma_{y}-k_{y} \sigma_{x}\right)$ of Eq. (1) becomes $K\left(k_{x} \sigma_{y-} k_{y} \sigma_{x}\right)+K^{\prime}\left(\varepsilon_{x x}+\varepsilon_{y y}\right)\left(k_{x} \sigma_{y-} k_{y} \sigma_{x}\right)$. Also, in Eq. (1), $E_{0}$ is renormalized to $E_{0}+E^{\prime}\left(\varepsilon_{x x}+\varepsilon_{y y}\right)$, thus explaining results regarding $\Gamma^{1}$ and $\Gamma^{2}$ in Fig. 3(a).

Let us now comment on possible applications of $\mathrm{LaWN}_{3}$ 's spin splittings and textures. First, note that cubic-in- $k$ and squared-in- $k$ terms are pivotal for reproducing spin textures around the $\Gamma$ and/or $L$ points, respectively. High-order spin splittings definitely have potential applications such as cubic Rashba-enhanced spin Hall transport of heavy holes [60]. Second, as shown in Figs. 2(e) and 2(f), the spin textures for two lower bands around the $L$ point are nearly momentum $(k)$ independent. Such quasipersistent spin textures [61] (promising for nondephasing spin transport $[61,62]$ ), together with large spin splitting, form another prospective aspect. Third, the discovery of different types of spin textures may promote exotic device applications as evidenced by, e.g., linear magnetoelectric effect driven by the "band splitting with vanishing spin polarizations" phenomenon [63]. We are also aware of interesting spin textures and mechanisms for Rashba spin splittings in multiferroic $\mathrm{BiCoO}_{3}$ with canted spins [64]. All of these aspects show the fundamental but also technological significance of obtaining types of spin textures and developing models to explain them.

\section{SUMMARY}

We have shown that the ferroelectric $R 3 c \mathrm{LaWN}_{3}$ is a promising material having a large spin splitting $\sim 2.7 \mathrm{eV} \AA$. We also identified the PODF-contributed spin splittings for bands containing both spin and orbital degrees of freedom. In particular, we emphasize the importance of the PODF--a unique feature of $2 n$-band ( $n \geqslant 2$, e.g., four-band and sixband) Hamiltonian-to achieve the enhanced spin splittings; this property should be sought and considered as a path to screen materials with large spin splittings. Moreover, the spin textures around the $L$ and $\Gamma$ points are too unusual to be reproducible by a simple two-band $\boldsymbol{k} \bullet \boldsymbol{p}$ theory. Our fourband $\boldsymbol{k} \bullet \boldsymbol{p}$ theories containing significant squared- and/or cubic-in- $k$ spin splitting terms are required to reproduce these textures. Finally, the energy levels, especially those around the $L$ point, can be tuned by tensile strain without diminishing their large spin splittings. These nonconventional spin textures, large splitting, and tunability of the energy levels around the $L$ point should make $\mathrm{LaWN}_{3}$ perovskite promising for spintronics. We thus hope that our findings will be verified by experiments.

\section{ACKNOWLEDGMENTS}

H.J.Z. (DFT simulations, analytical derivations) and L.B. (project leader) thank the Department of Energy, Office of Basic Energy Sciences, under Award No. DE-SC0002220. P.C. (parameterization of the models) acknowledges ONR Grant No. N00014-17-1-2818. C.P. (suggestions to this work during data analysis and organizing the work) acknowledges ARO Grant No. W911NF-16-1-0227. Y.Y. thanks the National Natural Science Foundation of China (Contract No. 11874207). H.J.Z. and L.B. thank Professor Hiroyuki Nakamura for his valuable suggestions. This work is also supported by Arkansas High Performance Computing Center and HPC resources of CALMIP (Allocation No. 2019/P1229).
[1] S. Picozzi, Front. Phys. 2, 10 (2014).

[2] J. Varignon, J. Santamaria, and M. Bibes, Phys. Rev. Lett. 122, 116401 (2019).

[3] H. Djani, A. C. Garcia-Castro, W.-Y. Tong, P. Barone, E. Bousquet, S. Picozzi, and P. Ghosez, npj Quantum Mater. 4, 51 (2019).

[4] L. G. D. da Silveira, P. Barone, and S. Picozzi, Phys. Rev. B 93, 245159 (2016).

[5] L. L. Tao and E. Y. Tsymbal, Nat. Commun. 9, 2763 (2018).

[6] D. D. Sante, P. Barone, R. Bertacco, and S. Picozzi, Adv. Mater. 25, 509 (2013).

[7] E. Plekhanov, P. Barone, D. Di Sante, and S. Picozzi, Phys. Rev. B 90, 161108(R) (2014).

[8] M. Kim, J. Im, A. J. Freeman, J. Ihm, and H. Jin, Proc. Natl. Acad. Sci. USA 111, 6900 (2014).

[9] L. L. Tao, T. R. Paudel, A. A. Kovalev, and E. Y. Tsymbal, Phys. Rev. B 95, 245141 (2017).
[10] D. Di Sante, P. Barone, A. Stroppa, K. F. Garrity, D. Vanderbilt, and S. Picozzi, Phys. Rev. Lett. 117, 076401 (2016).

[11] A. Stroppa, D. D. Sante, P. Barone, M. Bokdam, G. Kresse, C. Franchini, M.-H. Whangbo, and S. Picozzi, Nat. Commun. 5, 5900 (2014).

[12] A. Narayan, Phys. Rev. B 92, 220101(R) (2015).

[13] C. Autieri, P. Barone, J. Sławińska, and S. Picozzi, Phys. Rev. Mater. 3, 084416 (2019).

[14] R. Arras, J. Gosteau, H. J. Zhao, C. Paillard, Y. Yang, and L. Bellaiche, Phys. Rev. B 100, 174415 (2019).

[15] J. He, D. D. Sante, R. Li, X.-Q. Chen, J. M. Rondinelli, and C. Franchini, Nat. Commun. 9, 492 (2018).

[16] R. Sarmiento-Peréz, T. F. T. Cerqueira, S. Korbel, S. Botti, and M. A. L. Marques, Chem. Mater. 27, 5957 (2015).

[17] S. Körbel, M. A. L. Marques, and S. Botti, J. Mater. Chem. C 4, 3157 (2016). 
[18] Y.-W. Fang, C. A. J. Fisher, A. Kuwabara, X.-W. Shen, T. Ogawa, H. Moriwake, R. Huang, and C.-G. Duan, Phys. Rev. B 95, 014111 (2017).

[19] M. El-Batanouny and F. Wooten, Symmetry and Condensed Matter Physics: A Computational Approach (Cambridge University Press, New York, 2008).

[20] S. M. Young and C. L. Kane, Phys. Rev. Lett. 115, 126803 (2015).

[21] S. R. Bauers et al., Proc. Natl. Acad. Sci. USA 116, 14829 (2019).

[22] K. R. Talley et al., Adv. Electron. Mater. 5, 1900214 (2019).

[23] K. R. Talley, C. L. Perkins, D. R. Diercks, G. L. Brennecka, and A. Zakutayev, arXiv:2001.00633.

[24] See Supplemental Material at http://link.aps.org/supplemental/ 10.1103/PhysRevB.102.041203 for the developed $\boldsymbol{k} \bullet \boldsymbol{p}$ models, computational details, and some supplementary results (e.g., electronic structures, ferroelectric switching of spin textures) regarding $\mathrm{LaWN}_{3}$.

[25] P. E. Blöchl, Phys. Rev. B 50, 17953 (1994).

[26] H. T. Stokes, D. M. Hatch, and B. J. Campbell, FINDSYM, ISOTROPY Software Suite, iso.byu.edu.

[27] H. T. Stokes and D. M. Hatch, J. Appl. Crystallogr. 38, 237 (2005).

[28] M. I. Aroyo, J. M. Perez-Mato, C. Capillas, E. Kroumova, S. Ivantchev, G. Madariaga, A. Kirov, and H. Wondratschek, Z. Kristallogr. 221, 15 (2006).

[29] Y. Hinuma, G. Pizzi, Y. Kumagai, F. Oba, and I. Tanaka, Comput. Mater. Sci. 128, 140 (2017).

[30] A. Togo and I. Tanaka, arXiv:1808.01590.

[31] J. D. Hunter, Comput. Sci. Eng. 9, 90 (2007).

[32] http://pymatgen.org/.

[33] S. P. Ong, W. D. Richards, A. Jain, G. Hautier, M. Kocher, S. Cholia, D. Gunter, V. Chevrier, K. A. Persson, and G. Ceder, Comput. Mater. Sci. 68, 314 (2013).

[34] K. Momma and F. Izumi, J. Appl. Crystallogr. 44, 1272 (2011).

[35] https://materialsproject.org/.

[36] A. Jain, S. P. Ong, G. Hautier, W. Chen, W. D. Richards, S. Dacek, S. Cholia, D. Gunter, D. Skinner, G. Ceder, and K. A. Persson, APL Mater. 1, 011002 (2013).

[37] Wolfram Research, Inc., SystemModeler, Version 12.0, Champaign, IL (2019).

[38] G. Kresse and J. Furthmüller, Phys. Rev. B 54, 11169 (1996).

[39] G. Kresse and D. Joubert, Phys. Rev. B 59, 1758 (1999).

[40] G. F. Koster, J. D. Dimmock, R. G. Wheeler, and H. Statz, Properties of the Thirty-two Point Groups (M.I.T. Press, Cambridge, MA, 1963).
[41] http://cryst.ehu.es/.

[42] http://cryst.ehu.es/cryst/get_gen.html.

[43] https://www.materialscloud.org/work/tools/seekpath.

[44] J. P. Perdew, A. Ruzsinszky, G. I. Csonka, O. A. Vydrov, G. E. Scuseria, L. A. Constantin, X. Zhou, and K. Burke, Phys. Rev. Lett. 100, 136406 (2008).

[45] F.-X. Xiang, X.-L. Wang, M. Veldhorst, S.-X. Dou, and M. S. Fuhrer, Phys. Rev. B 92, 035123 (2015).

[46] M. Sakano, M. S. Bahramy, A. Katayama, T. Shimojima, H. Murakawa, Y. Kaneko, W. Malaeb, S. Shin, K. Ono, H. Kumigashira, R. Arita, N. Nagaosa, H. Y. Hwang, Y. Tokura, and K. Ishizaka, Phys. Rev. Lett. 110, 107204 (2013).

[47] K. Ishizaka, M. S. Bahramy, H. Murakawa, M. Sakano, T. Shimojima, T. Sonobe, K. Koizumi, S. Shin, H. Miyahara, A. Kimura, K. Miyamoto, T. Okuda, H. Namatame, M. Taniguchi, R. Arita, N. Nagaosa, K. Kobayashi, Y. Murakami, R. Kumai, Y. Kaneko, Y. Onose, and Y. Tokura, Nat. Mater. 10, 521 (2011).

[48] L. Schimka, J. Harl, and G. Kresse, J. Chem. Phys. 134, 024116 (2011).

[49] R. Winkler and U. Zülicke, Phys. Rev. B 82, 245313 (2010).

[50] Sz. Vajna, E. Simon, A. Szilva, K. Palotas, B. Ujfalussy, and L. Szunyogh, Phys. Rev. B 85, 075404 (2012).

[51] S. Bandyopadhyay, A. Paul, and I. Dasgupta, Phys. Rev. B 101, 014109 (2020).

[52] J. P. Perdew, K. Burke, and M. Ernzerhof, Phys. Rev. Lett. 77, 3865 (1996).

[53] S. L. Dudarev, G. A. Botton, S. Y. Savrasov, C. J. Humphreys, and A. P. Sutton, Phys. Rev. B 57, 1505 (1998).

[54] J. Sun, A. Ruzsinszky, and J. P. Perdew, Phys. Rev. Lett. 115, 036402 (2015).

[55] L. C. Lew Yan Voon and M. Willatzen, The kp Method (Springer Verlag, Berlin, 2009).

[56] H. J. Zhao, C. Xu, Y. Yang, W. Duan, X. M. Chen, and L. Bellaiche, J. Phys.: Condens. Matter 27, 485901 (2015).

[57] R. D. King-Smith and D. Vanderbilt, Phys. Rev. B 47, 1651(R) (1993).

[58] M. Ye and D. Vanderbilt, Phys. Rev. B 93, 134303 (2016).

[59] M. Bibes (private communication).

[60] J. Schliemann and D. Loss, Phys. Rev. B 71, 085308 (2005).

[61] J. Schliemann, J. C. Egues, and D. Loss, Phys. Rev. Lett. 90, 146801 (2003).

[62] J. D. Koralek, C. P. Weber, J. Orenstein, B. A. Bernevig, S.-C. Zhang, S. Mack, and D. D. Awschalom, Nature (London) 458, 610 (2009).

[63] K. Liu, W. Luo, J. Ji, P. Barone, S. Picozzi, and H. Xiang, Nat. Commun. 10, 5144 (2019).

[64] K. Yamauchi, P. Barone, and S. Picozzi, Phys. Rev. B 100, 245115 (2019). 